\title{
Effects of 1-year anti-TNF-a therapy on vascular function in rheumatoid arthritis and ankylosing spondylitis
}

\author{
Edit Végh ${ }^{1}$ - György Kerekes ${ }^{2}$ - Anita Pusztai ${ }^{1}$ - Attila Hamar ${ }^{1}$ - Szilvia Szamosi ${ }^{1}$. Andrea Váncsa ${ }^{1}$. Levente Bodoki ${ }^{1}$. \\ Lilla Pogácsás ${ }^{1} \cdot$ Fruzsina Balázs $^{1} \cdot$ Katalin Hodosi $^{1} \cdot$ Andrea Domján $^{1} \cdot$ Sándor Szántó ${ }^{1,3}$ • Zoltán Nagy ${ }^{1}$. \\ Zoltán Szekanecz ${ }^{1}$ - Gabriella Szücs ${ }^{1}$
}

Received: 26 October 2019 / Accepted: 10 December 2019 / Published online: 17 December 2019

(c) The Author(s) 2019

\begin{abstract}
Accelerated atherosclerosis, increased cardiovascular morbidity and mortality have been associated with rheumatoid arthritis (RA) and ankylosing spondylitis (AS). Vascular function, clinical and laboratory markers and the effects of anti-TNF therapy were assessed in arthritides. Fifty-three 53 patients including 36 RA patients treated with either etanercept (ETN) or certolizumab pegol and 17 AS patients treated with ETN were included in a 12-month follow-up study. Ultrasonography was performed to determine flow-mediated vasodilation (FMD), common carotid intima-media thickness (ccIMT) and arterial pulse-wave velocity (PWV) in all patients. All assessments were performed at baseline and 6 and 12 months after treatment initiation. A significant improvement of brachial artery FMD was observed after 6 months $(p=0.004)$. A tendency of FMD improvement was also observed after 12 months $(p=0.065)$. ccIMT did not change throughout the year. PWV significantly improved after 12 months $(p=0.034)$. Higher baseline ccIMT $(p=0.009)$ and PWV $(p=0.038)$ were associated with clinical non-response (cNR) versus response (cR) to biologics. Multiple analysis confirmed the association of baseline ccIMT with age $(p=0.003)$ and cNR $(p=0.009)$, as well as that of baseline PWV with age at diagnosis $(p=0.022)$ and current chest pain $(p=0.004)$. Treatment itself determined the 12-month changes in FMD $(p=0.020)$ and PWV $(p=0.007)$. In a mixed cohort of RA and AS patients, TNF inhibition improved or stabilized vascular pathophysiology. Inflammation may be associated with FMD, while, among others, cNR may influence vascular function.
\end{abstract}

Keywords Rheumatoid arthritis $\cdot$ Ankylosing spondylitis $\cdot$ Atherosclerosis $\cdot$ Cardiovascular disease $\cdot$ Flow-mediated vasodilation $\cdot$ Pulse-wave velocity $\cdot$ Carotid intima-media thickness

$\begin{array}{ll}\text { Abbreviations } \\ \text { ACPA } & \text { Anti-citrullinated protein antibody } \\ \text { AIx } & \text { Augmentation Index } \\ \text { AS } & \text { Ankylosing spondylitis } \\ \text { BASDAI } & \text { Bath Ankylosing Spondylitis Disease Activity } \\ & \text { Index } \\ \text { ccIMT } & \text { Common carotid intima-media thickness } \\ \text { CCP } & \text { Cyclic citrullinated peptide } \\ \text { cNR } & \text { Clinical non-response } \\ \text { cR } & \text { Clinical response } \\ \text { CRP } & \text { C-reactive protein } \\ \text { CV } & \text { Cardiovascular } \\ \text { CVD } & \text { Cardiovascular disease }\end{array}$

Zoltán Szekanecz

szekanecz.zoltan@med.unideb.hu

http://www.rheumatology.hu

Extended author information available on the last page of the article

$\begin{array}{ll}\text { CZP } & \text { Certolizumab pegol } \\ \text { DAS } & \text { Disease activity score } \\ \text { ETN } & \text { Etanercept } \\ \text { EULAR } & \text { European League Against Rheumatism } \\ \text { FMD } & \text { Flow-mediated vasodilation } \\ \text { PWV } & \text { Pulse-wave velocity } \\ \text { RA } & \text { Rheumatoid arthritis } \\ \text { RF } & \text { Rheumatoid factor } \\ \text { SC } & \text { Subcutaneous } \\ \text { TNF } & \text { Tumor necrosis factor }\end{array}$

\section{Introduction}

Arthritides, such as rheumatoid arthritis (RA) and ankylosing spondylitis (AS) have been associated with accelerated, inflammatory atherosclerosis, as well as increased cardiovascular $(\mathrm{CV})$ morbidity and mortality [1-4]. It is crucial 
to detect $\mathrm{CV}$ abnormalities early, possibly in the preclinical phase of CV disease (CVD) [1]. Indeed, non-invasive ultrasound-based techniques, in addition to clinical and laboratory biomarkers, may be suitable to assess preclinical vascular pathophysiology in RA and AS [1, 5]. Early endothelial dysfunction, overt atherosclerosis and increased arterial stiffness are indicated by abnormal endothelium-dependent, flow-mediated vasodilation (FMD) of the brachial artery, common carotid intima-media thickness (ccIMT) and carotid plaques, as well as arterial pulse-wave velocity (PWV), respectively $[2,5]$. These preclinical abnormalities predict the development of subsequent $\mathrm{CV}$ events in arthritides [1, 2,5].

Systemic inflammation associated with RA and AS is the major driver of atherosclerosis and CVD in these diseases [1]. Pro-inflammatory cytokines, such as tumour necrosis factor $\alpha(\mathrm{TNF}-\alpha)$ is highly involved in the pathogenesis of RA-related atherosclerosis [6]. It is not surprising that the control of inflammation by targeted therapies including TNF- $\alpha$ inhibitors may dampen atherosclerosis and may decrease $\mathrm{CV}$ morbidity and mortality in inflammatory arthritis [7-10], especially in patients who respond to TNF inhibition $[7,8]$. With respect to surrogate markers, anti-TNF biologics may improve or at least stabilize vascular morphology and function including FMD [7, 11-17], ccIMT [7, 13, 16, 18-20] and PWV [7, 21-25].

In this study, we wished to determine the effects of oneyear anti-TNF therapy on FMD, ccIMT and PWV. We also wished to determine the predictors of these parameters at baseline, as well as the determinants on one-year change in these parameters. This study may improve our understanding of vascular pathophysiology in RA and AS.

\section{Patients and methods}

\section{Patients}

Fifty-three patients with inflammatory arthritis (36 RA and $17 \mathrm{AS}$ ) selected for the initiation of anti-TNF therapy but unselected for CVD were enrolled in the study. Patient characteristics are seen in Table 1 . The cohort included 34 women and 19 men with mean age of $52.0 \pm 12.1$ (range 24-83) years. Mean disease duration was $8.5 \pm 7.9$ (range 1-44) years, while the mean age at diagnosis was $43.5 \pm 12.1$ (range 23-62) years. Exclusion criteria included untreated, unstable hypertension (blood pressure $>140 / 90 \mathrm{mmHg}$ ), diabetes mellitus, current inflammatory disease other than RA or AS, infectious disease or renal failure (serum creatinine $\geq 117 \mathrm{mmol} / \mathrm{l}$ ). None of the patients received aspirin, clopidogrel, heparin or warfarin or vasoactive drugs at the time of inclusion. Patients with active disease were recruited prior to initiating a biological therapy. All patients started on an anti-TNF therapy at baseline and received the same biological treatment at one year. Among the $36 \mathrm{RA}$ patients, 20 received etanercept (ETN) $50 \mathrm{mg} /$ week subcutaneous (SC) and 16 received certolizumab pegol (CZP) (400 $\mathrm{mg}$ at 0,2 and 4 weeks, and thereafter $200 \mathrm{mg}$ twice weekly SC). Altogether 18 RA patients were treated with ETN and 13 with CZP in combination with methotrexate (MTX). The other

Table 1 Patient characteristics

\begin{tabular}{llll}
\hline & RA & AS & Total \\
\hline$n$ & 36 & 17 & 53 \\
Female:male & $31: 5$ & $3: 14$ & $34: 19$ \\
Age (mean \pm SEM)(range), years & $55.9 \pm 9.8(35-83)$ & $43.6 \pm 12.4(24-72)$ & $52.0 \pm 12.1(24-83)$ \\
Disease duration (mean \pm SEM) (range), years & $9.1 \pm 8.3(1-44)$ & $7.2 \pm 7.0(1-26)$ & $8.5 \pm 7.9(1-44)$ \\
Age at diagnosis & $47.0 \pm 8.7(28-62)$ & $36.4 \pm 11.6(23-50)$ & $43.5 \pm 12.1(23-62)$ \\
Smoking (current) & 7 & 7 & 14 \\
Positive CV history & 8 & 1 & 9 \\
Current, stable hypertension & 3 & 0 & 3 \\
Diabetes mellitus & 1 & 0 & 1 \\
Periodontitis (current) & 10 & 5 & 15 \\
Tooth loss (current) & 14 & 7 & 21 \\
Current low-dose Corticosteroids & 12 & 2 & 14 \\
RF positivity, $n(\%)$ & $26(72)$ & - & - \\
ACPA positivity, $n(\%)$ & $21(58)$ & - & - \\
DAS28 (baseline) (mean \pm SEM) & $5.00 \pm 0.86$ & - & - \\
BASDAI (baseline) (mean \pm SEM) & - & $5.79 \pm 1.19$ & - \\
Treatment (ETN, CZP) & $20 \mathrm{ETN}, 16 \mathrm{CZP}$ & $17 \mathrm{ETN}$ & $37 \mathrm{ETN}, 16 \mathrm{CZP}$ \\
Biologic in combination with MTX & $18 \mathrm{ETN}, 13 \mathrm{CZP}$ & - & $18 \mathrm{ETN}, 13 \mathrm{CZP}$ \\
\hline
\end{tabular}


patients received monotherapy. All 17 AS patients received $50 \mathrm{mg} /$ week ETN monotherapy SC. RA patients did not take DMARDs other than MTX. Altogether $12 \mathrm{RA}$ and 2 AS patients currently took low-dose $(<6 \mathrm{mg} /$ day $)$ methylprednisolone (Table 1).

Disease activity was determined by DAS28 and BASDAI in RA and AS, respectively. Clinical responder (cR vs cNR) status was determined after 12 months of treatment with either ETN or CZP by the EULAR response criteria originally reported by Van Gestel et al. [26] and described by us before [27].

The study was approved by the Hungarian Scientific Research Council Ethical Committee (Approval no. 14804-2/2011/EKU). Written informed consent was obtained from each patient and assessments were carried out according to the Declaration of Helsinki.

\section{Clinical assessment}

First, a detailed medical history was taken. We inquired for the history of CVD, as well as current smoking, the experience of chest pain resembling angina pectoris, hypertension and diabetes mellitus during the last 2 years prior to the start of this study by a questionnaire (Table 1). Further clinical assessments including physical examination were performed at baseline, and after 3, 6 and 12 months of therapy. At baseline, RA patients had a mean DAS28 of $5.00 \pm 0.86$, while AS patients exerted mean BASDAI of $5.79 \pm 1.19$ (Table 1).

\section{Laboratory measurements}

Serum high sensitivity $\mathrm{C}$ reactive protein (hsCRP; nor$\mathrm{mal}: \leq 5 \mathrm{mg} / \mathrm{l}$ ) and $\mathrm{IgM}$ rheumatoid factor (RF; nor$\mathrm{mal}: \leq 50 \mathrm{IU} / \mathrm{ml}$ ) were measured by quantitative nephelometry (Cobas Mira Plus-Roche), using CRP and RF reagents (both Dialab). ACPA (anti-CCP) autoantibodies were detected in serum samples using a second-generation Immunoscan-RA CCP2 ELISA test (Euro Diagnostica; nor$\mathrm{mal}: \leq 25 \mathrm{IU} / \mathrm{ml})$. The assay was performed according to the manufacturer's instructions.

\section{Assessment of vascular physiology by ultrasound}

Brachial artery FMD was assessed as described before [2, $28,29]$. In brief, ultrasound examination was performed on the right arm using $10 \mathrm{MHz}$ linear array transducer (ultrasound system: HP Sonos 5500) by a single trained sonographer after $30 \mathrm{~min}$ rest in a temperature-controlled room (basal value for FMD). A B-mode longitudinal section was obtained from the brachial artery above the antecubital fossa. To assess FMD, reactive hyperaemia was induced by the release of a pneumatic cuff around the forearm inflated to suprasystolic pressure for $4.5 \mathrm{~min}$. After deflation the maximal flow velocity and the arterial diameter was $90 \mathrm{~s}$ long continuously recorded. Flow velocities, the baseline diameter, as well as FMD were ECG gated and detected offline. FMD values were expressed as \% change from baseline (resting) value.

The ccIMT measurements were carried out as described before $[2,29,30]$. Briefly, a duplex ultrasound system (HP Sonos 5500, $10 \mathrm{MHz}$ linear array transducer) was used to assess the common carotid arteries by a single observer. Longitudinal high-resolution B-mode ultrasound scan was employed over both right and left common carotid arteries and were R-synchronized and recorded. The offline measurements were performed $1 \mathrm{~cm}$ proximal to the carotid bulb in the far wall. ccIMT was defined as the distance between the first and second echogenic lines from the lumen taking the average of 10 measurements on both sides. ccIMT values were expressed in $\mathrm{mm}$.

With respect to arterial stiffness, PWV was calculated automatically by a TensioClinic arteriograph system (Tensiomed Ltd, Budapest, Hungary) as the quotient of the distance between the jugular fossa and symphysis as described before [29, 31]. If an artery is elastic, PWV is low. With decreased arterial elasticity, PWV rises. The arteriograph assesses this parameter from the oscillometric data obtained from the $35 \mathrm{mmHg}$ suprasystolic pressure of the brachial artery. To obtain reproducible results, the patient had to rest in a supine position for at least $10 \mathrm{~min}$ before the assessment in a quiet room. PWV is expressed in $\mathrm{m} / \mathrm{s}$. Reproducibility of the three techniques expressed in intraclass correlation is included in the Statistical analysis section.

\section{Statistical analysis}

Statistical analysis was performed using SPSS version 22.0 (IBM) software. Data are expressed as the mean \pm SD for continuous variables and percentages for categorical variables. The distribution of continuous variables was evaluated by Kolmogorov-Smirnov test. Independent and paired twotailed $t$ test were used assessed the differences. Nominal variables were compared between groups using the chi-squared or Fisher's exact test, as appropriate. Correlations were determined by Pearson's analyses. Univariate and multiple regression analysis using the stepwise method were applied to investigate independent associations between angiogenic biomarkers (dependent variables) and other clinical, laboratory and imaging parameters (independent variables). The $\beta$ standardized linear coefficients showing linear correlations between two parameters were determined. The $B(+95 \%$ CI) regression coefficient indicated independent associations between dependent and independent variables during changes. Repeated measures analysis of variance (RMANOVA) was performed to determine the additional effects of multiple parameters on changes of vascular imaging 
markers between baseline and 12 months. The dependent variables were FMD, ccIMT and PWV. Partial $\eta^{2}$ is given as an indicator of effect size, with values of 0.01 suggesting small, 0.06 medium and 0.14 large effects. Three related samples were compared by the Friedman test. The reliability was tested by inter-item correlation and intraclass correlation (ICC). With respect to the FMD, ccIMT and PWV tests, ICC $=0.470 ; F$ test value: $1.887 ; p=0.001$. The power was estimated with G-Power software. $p$ values $<0.05$ were considered significant.

\section{Results}

\section{Effects of anti-TNF therapy on clinical activity, inflammation and clinical response in RA and AS}

In RA ( $n=36)$, one-year ETN or CZP therapy significantly improved DAS28 (12 months: $3.02 \pm 0.96$; baseline: $5.00 \pm 0.86 ; p<0.001$ ) (data not shown). Similarly, in AS $(n=17)$, BASDAI significantly decreased from $5.79 \pm 1.19$ (baseline) to $1.86 \pm 1.04$ after 12 months of ETN treatment $(p<0.001)$ (data not shown).

With respect to inflammation, in the mixed arthritis cohort, CRP significantly decreased as early as after
3 months of treatment $(7.57 \pm 12.02 \mathrm{mg} / \mathrm{l})$ compared to baseline $(14.88 \pm 17.09 \mathrm{mg} / \mathrm{l} ; p<0.001)$. This effect was even more pronounced after 6 months $(6.79 \pm 9.52 \mathrm{mg} / \mathrm{l}$; $p<0.001)$ and 12 months of therapy $(6.49 \pm 7.60 \mathrm{mg} / \mathrm{l}$; $p<0.001$ ) (data not shown).

As described above, $\mathrm{cR}$ and $\mathrm{cNR}$ status of the patients was also determined [26, 27]. Altogether 34 patients (64\%) were $\mathrm{cR}$ and 19 (36\%) were cNR (data not shown).

\section{Effects of TNF inhibition on vascular pathophysiology}

Although, as mentioned in the Discussion, FMD, ccIMT and PWV do not have "normal values", baseline FMD was lower, while ccIMT and PWV were higher in RA compared to AS reflecting more pronounced pathology in RA compared to AS (data not shown).

In the mixed cohort of 53 arthritis patients, brachial artery FMD significantly improved after 6 months of anti$\mathrm{TNF}$ therapy $(10.01 \pm 5.26 \%)$ versus baseline $(7.67 \pm 4.40 \%$; $p=0.004$ ) (Fig. 1). This improvement was also observed, however, non-significantly, after 12 months $(9.70 \pm 4.91 \%$; $p=0.065$ ) (Fig. 1). ccIMT remained stable showing no significant progression after 6 months $(0.59 \pm 0.11 \mathrm{~mm}$; $p=0.09)$ and 12 months $(0.59 \pm 0.12 \mathrm{~mm} ; p=0.240)$

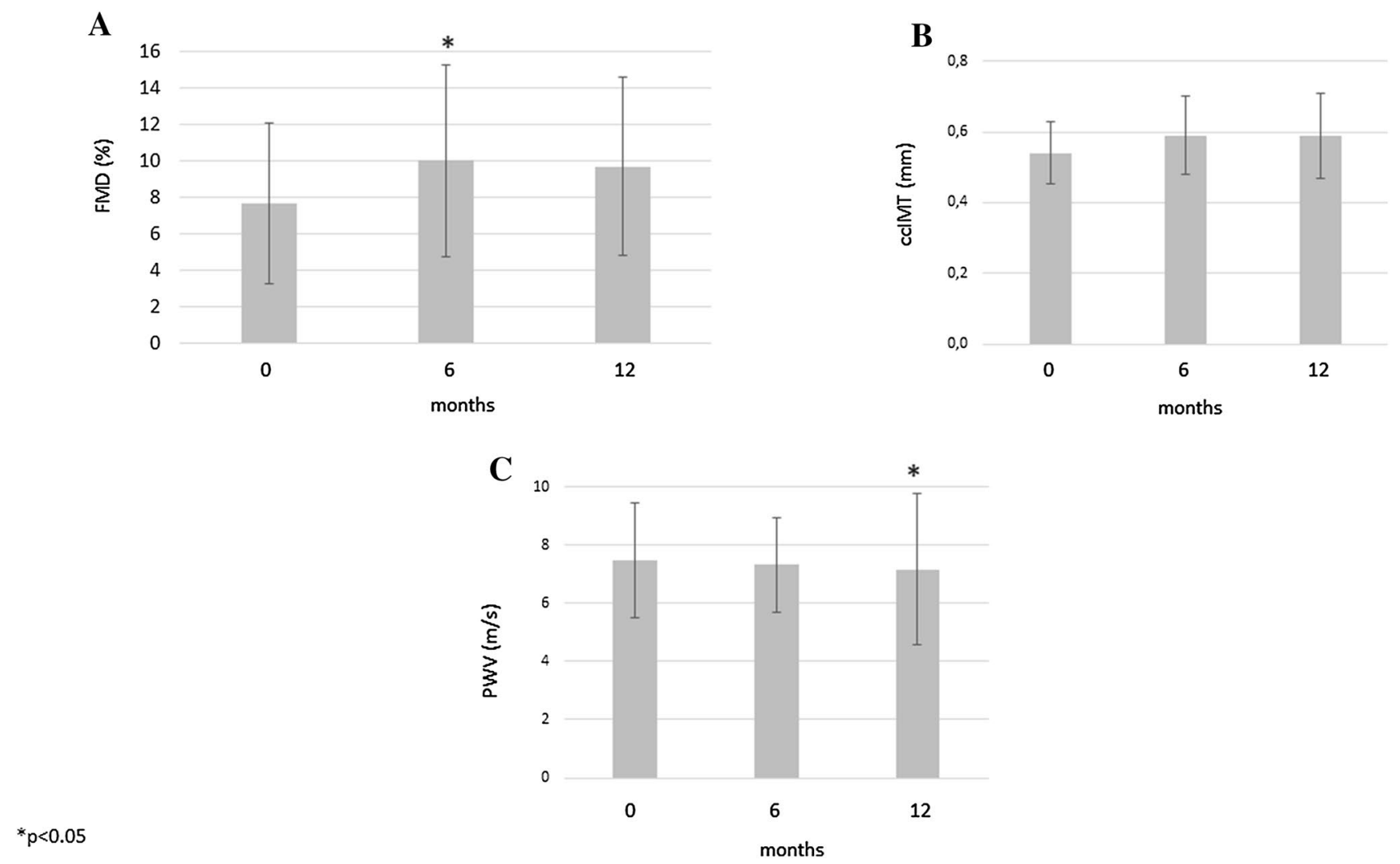

Fig. 1 Effects of 1-year anti-TNF therapy on FMD (a), ccIMT (b) and PWV (c) in RA and AS. Asterisks indicate significant changes $(p<0.05)$ 
compared to baseline $(0.540 \pm 0.087 \mathrm{~mm})$ (Fig. 1) PWV reflecting arterial stiffness slightly improved after 6 months $(7.32 \pm 1.63 \mathrm{~m} / \mathrm{s} ; p=0.866)$ versus baseline $(7.48 \pm 1.98 \mathrm{~m} / \mathrm{s})$. This improvement was significant after 12 months $(7.17 \pm 2.59 \mathrm{~m} / \mathrm{s} ; p=0.034)$ (Fig. 1). We also assessed the effects of ETN vs CZP separately and we did not find any differences in FMD, ccIMT or PWV at baseline and after 6 and 12 months (data not shown).

\section{Correlations of markers of vascular pathophysiology with clinical response and other parameters}

When nominal variables were evaluated, patients with a history of CVD had significantly higher ccIMT after 6 months $(0.60 \pm 0.07 \mathrm{~mm}$ vs $0.55 \pm 0.09 \mathrm{~mm} ; p=0.045)$ and after 12 months $(0.61 \pm 0.12 \mathrm{~mm}$ vs $0.54 \pm 0.10 \mathrm{~mm} ; p=0.033)$, as well as higher PWV at baseline $(8.34 \pm 2.29 \mathrm{~m} / \mathrm{s}$ vs $6.62 \pm 1.13 \mathrm{~m} / \mathrm{s} ; p=0.005)$ and after 6 months $(7.94 \pm 1.86 \mathrm{~m} / \mathrm{s}$ vs $6.66 \pm 1.23 \mathrm{~m} / \mathrm{s} ; p=0.012)$ compared to patients with negative CVD history (data not shown). Moreover, patients currently experiencing chest pain also had significantly higher baseline PWV $(8.64 \pm 2.47 \mathrm{~m} / \mathrm{s}$ vs $7.59 \pm 2.00 \mathrm{~m} / \mathrm{s} ; p=0.017)$ versus those who never had chest pain previously (data not shown). Patients with hypertension had higher 12-month ccIMT $(0.62 \pm 0.11 \mathrm{~mm}$ vs $0.55 \pm 0.11 \mathrm{~mm} ; p=0.050)$ and baseline PWV $(8.36 \pm 2.24 \mathrm{~m} / \mathrm{s}$ vs $6.88 \pm 1.56 \mathrm{~m} / \mathrm{s} ; p=0.016)$ compared to those with normal blood pressure (data not shown).

When comparing vascular pathophysiology with clinical responsiveness, $\mathrm{cNR}$ patients had significantly higher baseline ccIMT $(0.60 \pm 0.08 \mathrm{~mm}$ vs $0.52 \pm 0.08 \mathrm{~mm} ; p=0.009)$; 6 -month ccIMT $(0.64 \pm 0.14 \mathrm{~mm}$ vs $0.56 \pm 0.09 \mathrm{~mm}$; $p=0.023)$ and baseline PWV $(8.47 \pm 2.62 \mathrm{~m} / \mathrm{s}$ vs $7.08 \pm 1.55 \mathrm{~m} / \mathrm{s} ; p=0.038$ ) compared to $\mathrm{cR}$ patients (data not shown).

When vascular imaging parameters were correlated with each other, baseline and 12-month ccIMT correlated with baseline and 12-month PWV, respectively (data not shown).

With respect to simple correlations of vascular imaging and other parameters, baseline and 12-month ccIMT, as well as 12-month PWV significantly correlated with age. Baseline ccIMT and PWV, as well as 12-month ccIMT and PWV also correlated with age at diagnosis of RA. Baseline FMD, as well as baseline and 12-month PWV showed a positive correlation with baseline CRP (Table 2).

Results of the univariate and multiple analyses are indicated in Table 3. In the univariate analysis of the mixed $\mathrm{RA}+\mathrm{AS}$ population, baseline FMD was determined by baseline CRP $(p=0.040)$. The predictors of baseline ccIMT were age, age at diagnosis and cNR vs cR status $(p<0.05)$. ccIMT after 12 months of treatment was determined by age, age at diagnosis, as well as the history of CVD $(p<0.05)$. Age at diagnosis, history of CVD and current hypertension,
Table 2 Significant correlations between markers of vascular pathophysiology and other parameters in the RA + AS population

\begin{tabular}{lllll}
\hline & Age & Age at diagnosis & CRP-0 & CRP-12 \\
\hline FMD-0 & & & $R=0.318$ & \\
& & & $p=0.040$ & \\
ccIMT-0 & $R=0.449$ & $R=0.350$ & & $R=0.403$ \\
& $p=0.003$ & $p=0.023$ & & $p=0.008$ \\
ccIMT-12 & $R=0.477$ & $R=0.399$ & & \\
& $p=0.001$ & $p=0.005$ & & \\
PWV-0 & & $R=0.351$ & $R=0.416$ & \\
& & $p=0.023$ & $p=0.020$ & \\
PWV-12 & $R=0.571$ & $R=0.503$ & $R=0.411$ & \\
& $p<0.001$ & $p<0.001$ & $p=0.022$ & \\
\hline
\end{tabular}

See text for abbreviations and explanations

current chest pain and cNR vs cR status predicted baseline PWV $(p<0.05)$. Finally, 12-month PWV was determined by age and age at diagnosis $(p<0.001)$ (Table 3$)$.

The multiple analysis of the RA + AS population confirmed the significant association of baseline ccIMT with age $(p=0.003)$ and cNR vs cR status $(p=0.009)$. ccIMT after 12 months of treatment was also associated with age $(p<0.001)$. Baseline PWV was determined by age at diagnosis $(p=0.022)$ and by current chest pain $(p=0.004)$, while 12 -month PWV showed association with age $(p<0.001)$ (Table 3).

When RA and AS patients were analyzed separately, according to the univariate analysis, the disease itself (RA vs AS) had a significant effect on ccIMT and PWV. However, in the multiple analysis we could not demonstrate this, therefore, the disease itself is not an independent prognostic factor for vascular pathophysiology (Table 3).

Finally, RM-ANOVA analysis was performed in the $\mathrm{RA}+\mathrm{AS}$ mixed population to assess the determinants of 12-month changes in vascular imaging parameters. AntiTNF treatment itself determined the changes of FMD $(p=0.020)$, ccIMT $(p=0.024)$ and PWV $(p=0.007)$ from baseline to 12-months. In addition, treatment exerted combined effects with $\mathrm{cR}(p=0.045)$ on changes in FMD. Moreover, treatment and age had a combined effect of changes in PWV $(p=0.003)$ (Table 4).

\section{Discussion}

First, as expected, anti-TNF therapy was clinically effective in both RA and AS as indicated by significant decreases of DAS28 and BASDAI, respectively. Altogether two-third of the patients responded to treatment $(\mathrm{cR})$.

Ultrasound-based imaging may enhance risk CV stratification in RA and AS [2, 3, 5, 20, 29, 32, 33] and, as also 
Table 3 Univariate and multiple analysis of determinants of vascular pathophysiology

\begin{tabular}{|c|c|c|c|c|c|c|c|c|c|}
\hline \multirow[t]{2}{*}{ Dependent variable } & \multirow[t]{2}{*}{ Independent variable } & \multicolumn{4}{|c|}{ Univariate analysis } & \multicolumn{4}{|c|}{ Multiple analysis } \\
\hline & & $B$ & CI $95 \%$ & $\beta$ & $p$ & $B$ & CI $95 \%$ & $\beta$ & $p$ \\
\hline FMD-0 & CRP-0 & 0.076 & $0.004-0.148$ & 0.318 & 0.040 & & & & \\
\hline \multirow[t]{4}{*}{ ccIMT-0 } & Age & 0.003 & $0.001-0.005$ & 0.449 & 0.003 & 0.003 & $0.001-0.005$ & 0.417 & 0.003 \\
\hline & Age at diagnosis & 0.003 & $0-0.005$ & 0.350 & 0.023 & & & & \\
\hline & RA vs AS & 0.083 & $0.033-0.133$ & 0.469 & 0.002 & & & & \\
\hline & $\mathrm{cNR}$ vs $\mathrm{cR}$ & 0.076 & $0.020-0.132$ & 0.400 & 0.009 & 0.069 & $0.019-0.120$ & 0.363 & 0.009 \\
\hline \multirow[t]{4}{*}{ ccIMT-12 } & Age & 0.005 & $0.002-0.007$ & 0.477 & 0.001 & 0.005 & $0.002-0.007$ & 0.526 & $<0.001$ \\
\hline & Age at diagnosis & 0.004 & $0.001-0.007$ & 0.399 & 0.005 & & & & \\
\hline & RA vs AS & 0.072 & $0.003-0.142$ & 0.292 & 0.042 & & & & \\
\hline & CVD (history) & 0.071 & $0.006-0.136$ & 0.314 & 0.033 & & & & \\
\hline \multirow[t]{6}{*}{ PWV-0 } & Age at diagnosis & 0.062 & $0.009-0.114$ & 0.351 & 0.023 & 0.056 & $0.008-0.104$ & 0.321 & 0.022 \\
\hline & RA vs AS & 1.548 & $0.357-2.738$ & 0.384 & 0.012 & & & & \\
\hline & CVD (history) & 1.715 & $0.589-2.841$ & 0.438 & 0.004 & & & & \\
\hline & Chest pain (current) & 1.803 & $0.630-2.976$ & 0.441 & 0.003 & 1.708 & $0.595-2.822$ & 0.418 & 0.004 \\
\hline & Hypertension (current) & 1.481 & $0.296-2.665$ & 0.371 & 0.016 & & & & \\
\hline & $\mathrm{cNR}$ vs $\mathrm{cR}$ & 1.391 & $0.078-2.704$ & 0.321 & 0.038 & & & & \\
\hline \multirow[t]{3}{*}{ PWV-12 } & Age & 0.122 & $0.070-0.173$ & 0.571 & $<0.001$ & 0.122 & $0.070-0.173$ & 0.571 & $<0.001$ \\
\hline & Age at diagnosis & 0.120 & $0.059-0.180$ & 0.503 & $<0.001$ & & & & \\
\hline & RA vs AS & 2.181 & $0.733-3.629$ & 0.404 & 0.004 & & & & \\
\hline
\end{tabular}

See text for abbreviations and explanations

Table 4 Results of RM-ANOVA for changes from baseline to 12 months in vascular imaging parameters in the RA+AS mixed population

\begin{tabular}{lllll}
\hline Variable & Effect & $F$ & $p$ & Partial $\eta^{2}$ \\
\hline FMD & Treatment & 4.179 & 0.020 & 0.122 \\
& Treatment $\times$ cR & 3.263 & 0.045 & 0.098 \\
ccIMT & Treatment & 3.958 & 0.024 & 0.102 \\
PWV & Treatment & 5.993 & 0.007 & 0.130 \\
& Treatment $\times$ age & 5.558 & 0.003 & 0.122 \\
\hline
\end{tabular}

recommended by EULAR, may be used for this purpose [1]. Ultrasound may have higher value during the assessment of CV risk than coronary calcium content determination [32]. There are no "normal values" for FMD, ccIMT and PWV in RA and AS due to the heterogeneity of patient populations and methodology. However, in various studies, FMD was 4.6-7.7\% in active, 8.6-13.5\% in anti-TNF treated arthritis patients and $8.3-14.9 \%$ in healthy controls $[2,3,12,14$, 15]. Similarly, ccIMT was $0.63-0.76 \mathrm{~mm}, 0.62-0.68 \mathrm{~mm}$ and $0.54-0.62 \mathrm{~mm}$ in active, biologic-treated patients and controls, respectively $[2,3,7,13,33,34]$. In a meta-analysis from 22 studies on 1384 RA patients and 1147 controls, the mean ccIMT values were 0.71 and $0.62 \mathrm{~mm}$, respectively [34]. Moreover, when RA patients with and without CV events were compared, most CV events occurred in patients with ccIMT $>0.91$ [33]. Finally, PWV was $8.3-8.6 \mathrm{~m} / \mathrm{s}$,
$7.5-7.7 \mathrm{~m} / \mathrm{s}$ and $7.5-8.0 \mathrm{~m} / \mathrm{s}$ in active, anti-TNF-treated patients and controls, respectively [3, 7, 22, 23]. In a recent meta-analysis of 10 studies and 208 patients, anti-TNF treatment improved PWV by a mean $0.53 \mathrm{~m} / \mathrm{s}$ [21].

The magnitude of FMD [7, 11-17], ccIMT [7, 13, 16, 18-20] and PWV values [7, 21-25] before and after treatment was similar to previously reported ones. TNF inhibition resulted in a transiently significant improvement in FMD by 6 months. A tendency for improvement was also observed after one year. Biologics may inhibit the development and progression of atherosclerosis and lower the incidence of CV events in arthritides [7-10]. In a number of studies, biologics, mostly infliximab, resulted in an increase in FMD [11-16, 35] which, in few studies, was transient [14]. There were only a few long-term studies lasting more than one year [12]. Improvement in FMD was usually associated with clinical response to biologics [12]. ccIMT did not change during the course of biological therapy. Similar results were published by some other groups in RA and AS $[13,16,19]$. One group reported improvement of ccIMT upon treatment with various biologics after one year [18]. We found that ccIMT may improve upon anti-TNF therapy in early RA [35]. Again, the improvement of carotid atherosclerosis was associated with clinical efficacy [18]. Without treatment, carotid atherosclerosis may progress over time in arthritides [19]. PWV significantly decreased by 12 months compared to baseline. Some other investigators also reported improvement of PWV upon anti-TNF therapy in RA [21, 
22]. We previously reported that TNF inhibition improved PWV in early RA [35]. Others found no such improvement, especially long-term [23, 24]. One group that found no change in stiffness assessed Augmentation Index (AIx) and not PWV [25]. However, a recent meta-analysis of 10 studies has also suggested the beneficial effects of biologics on arterial stiffness [21]. Interestingly, while there have been some reports on the effects of ETN on these parameters $[12,16,17]$, we did not find such data on CZP. Yet, CZP was found to alter endothelial cell gene expression including cell adhesion molecules [36] and attenuate the inflammatory state [36], activation and adhesion of endothelial cells [37].

Among vascular imaging markers, ccIMT and PWV correlated with each other both at baseline and after 12 months. We have previously found correlations between carotid atherosclerosis and stiffness [29], while other groups did not [38]. It is possible that ccIMT and PWV are not independently associated with each other and some other factors may be involved in this association [38].

Both higher ccIMT and PWV correlated with the history of CVD and current hypertension. Indeed, both carotid atherosclerosis and arterial stiffness are associated with CVD $[5,33]$. Moreover, as hypertension correlated with 12-month ccIMT, high blood pressure may aggravate consequent carotid atherosclerosis. It is also important, that early, nonradiographic SpA was not associated with increased ccIMT indicating that there may be a window of opportunity here [39]. As baseline PWV correlated with most parameters including $\mathrm{CV}$ history, current chest pain, hypertension and cR (Table 2), assessing arterial stiffness may be a good screening technique. These instruments are more simple than assessing FMD or ccIMT in routine clinical care [5, 31].

Clinical response to biologics may be associated with vascular pathophysiology. In this study, cNR patients had significantly higher baseline and 6-month ccIMT, as well as baseline PWV. cNR patients may reflect a more severe, difficult-to-treat subset of arthritis patients [40]. Sustained inflammation and clinical activity associated with clinical non-response to treatment drives accelerated atherosclerosis in RA and AS [1-3, 8]. Indeed, the reduction of myocardial infarction risk was observed mainly in anti-TNF responders [8].

Baseline FMD and baseline PWV correlated with CRP underscoring the effects of systemic inflammation and acute phase reactants on vascular pathophysiology [1,2]. It may also be relevant that baseline CRP correlated with 12-month PWV. Higher CRP at baseline may drive vascular pathophysiology resulting in higher arterial stiffness after one year, despite anti-TNF therapy. These results were confirmed by the univariate analysis.

The multiple analysis confirmed the association of baseline ccIMT with age and clinical non-response. Age was also a predictor of 12-month ccIMT. Age also determined baseline and 12-month PWV. Indeed, age correlated with carotid atherosclerosis in some other studies [2, 29]. We also mentioned that the improvement of carotid atherosclerosis was associated with clinical efficacy in other studies [18]. Baseline PWV was determined by current chest pain also supporting the influence of arterial stiffness on CVD [41]. Moreover, among stiffness parameters, PWV was a better predictor of CVD than augmentation index (AIx) [41], therefore, the assessment of PWV rather than that of AIx is recommended in arthritis patients [5, 29]. When RA and AS patients were analyzed separately, in the univariate analysis, the disease itself had an effect on atherosclerosis and stuffness. However, the multiple analysis did not confirm this. Therefore, the disease type may not be an independent predictor of vascular pathology.

In the RM-ANOVA analysis, TNF inhibition itself determined one-year changes in FMD and PWV. As described above, anti-TNF agents exert beneficial effects on endothelial function and arterial stiffness in numerous studies (reviewed in [7]]. FMD clearly, although sometimes transiently improves in most studies [7, 11-13, 15, 16], while PWV, in this study and some others, shows long-term improvement [7, 17, 21, 22]. In addition, the treatment itself exerted combined effects with $\mathrm{cR}$ on changes in FMD. Thus, not only the treatment itself but also $\mathrm{cR}$ may predict FMD changes. As discussed above, improvement in FMD was associated with cR to biologics in other studies [12]. Clinical efficacy of a TNF inhibitor is usually assessed after the first 12 weeks and FMD is also the earliest indicator of vascular pathophysiology [7, 28]. Moreover, anti-TNF treatment had a more pronounced effect on the occurrence of CVD in responders [8]. Finally, treatment and age had a combined effect on changes in PWV. Age, also in this study, is an important determinant of baseline ccIMT and PWV [2, 7, 29].

Our study may certainly have some limitations. The relatively small study sample may have obscured potentially significant results. In addition, patients with potentially positive history of CV disease were also included. RA and AS patients were not analyzed separately due to the relatively small number of patients.

In conclusion, one-year anti-TNF treatment significantly but transiently improved FMD improved PWV and stabilized ccIMT in a mixed cohort of RA and AS patients. This was accompanied by clinical responses to biologics. Based on simple correlation analysis, the assessment of arterial stiffness may be a suitable screening method. Systemic inflammation indicated by CRP may be a determinant of FMD, an early indicator of vascular pathophysiology. On the other hand, age, history of CVD, hypertension or chest pain may rather be associated with arterial stiffness and over carotid atherosclerosis. Biologic treatment itself, with or without other factors, determine FMD and PWV changes 
over 12 months. Ultrasound-based, non-invasive techniques, as also recommended by EULAR [1] exert additional value in determining $\mathrm{CV}$ burden and in monitoring the effects of anti-TNF agents on vascular pathophysiology in relation to clinical efficacy.

Acknowledgements Open access funding provided by University of Debrecen (DE). This research was supported by the European Union and the State of Hungary and co-financed by the European Social Fund in the framework of TAMOP-4.2.4.A/2-11/1-2012-0001 'National Excellence Program'(Z. S.); by the European Union grants GINOP2.3.2-15-2016-00015 and GINOP-2.3.2-15-2016-00050 (Z. S.); and by the Pfizer Investigator Initiated Research Grant no. WS1695414 (Z.S.).

Author contributions EV: data acquisition and interpretation. GK: study design, data acquisition and interpretation. AP: data analysis and acquisition. AH: data analysis and acquisition. SS1: study design, data analysis. AV: data analysis. LB: data analysis and acquisition. LP: data acquisition. FB: data acquisition. KH: data analysis and interpretation. SS2: study design, data interpretation. ZN: data analysis and acquisition. ZS: head of study group, data analysis, draft writing, supervision, study design. GS: study coordinator, data analysis, supervision, study design. All co-authors take full responsibility for the integrity of the study and all parts of the manuscripts.

\section{Compliance with ethical standards}

Conflict of interest The authors declare that they do not have any competing interests.

Ethics approval The study was approved by the Hungarian Scientific Research Council Ethical Committee (Approval no. 14804-2/2011/ EKU).

Informed consent Written informed consent was obtained from each patient and assessments were carried out according to the Declaration of Helsinki.

Open Access This article is licensed under a Creative Commons Attribution 4.0 International License, which permits use, sharing, adaptation, distribution and reproduction in any medium or format, as long as you give appropriate credit to the original author(s) and the source, provide a link to the Creative Commons licence, and indicate if changes were made. The images or other third party material in this article are included in the article's Creative Commons licence, unless indicated otherwise in a credit line to the material. If material is not included in the article's Creative Commons licence and your intended use is not permitted by statutory regulation or exceeds the permitted use, you will need to obtain permission directly from the copyright holder. To view a copy of this licence, visit http://creativecommons.org/licenses/by/4.0/.

\section{References}

1. Agca R, Heslinga SC, Rollefstad S, Heslinga M, McInnes IB, Peters MJ et al (2017) Eular recommendations for cardiovascular disease risk management in patients with rheumatoid arthritis and other forms of inflammatory joint disorders: 2015/2016 update. Ann Rheum Dis 76:17-28

2. Kerekes G, Szekanecz Z, Der H, Sandor Z, Lakos G, Muszbek L et al (2008) Endothelial dysfunction and atherosclerosis in rheumatoid arthritis: a multiparametric analysis using imaging techniques and laboratory markers of inflammation and autoimmunity. J Rheumatol 35:398-406

3. Bodnar N, Kerekes G, Seres I, Paragh G, Kappelmayer J, Nemethne ZG et al (2011) Assessment of subclinical vascular disease associated with ankylosing spondylitis. J Rheumatol 38:723-729

4. Castaneda S, Nurmohamed MT, Gonzalez-Gay MA (2016) Cardiovascular disease in inflammatory rheumatic diseases. Best Pract Res Clin Rheumatol 30:851-869

5. Kerekes G, Soltesz P, Nurmohamed MT, Gonzalez-Gay MA, Turiel M, Vegh E et al (2012) Validated methods for assessment of subclinical atherosclerosis in rheumatology. Nat Rev Rheumatol 8:224-234

6. Choy E, Ganeshalingam K, Semb AG, Szekanecz Z, Nurmohamed M (2014) Cardiovascular risk in rheumatoid arthritis: recent advances in the understanding of the pivotal role of inflammation, risk predictors and the impact of treatment. Rheumatology (Oxford) 53:2143-2154

7. Szekanecz Z, Kerekes G, Soltesz P (2009) Vascular effects of biologic agents in ra and spondyloarthropathies. Nat Rev Rheumatol 5:677-684

8. Dixon WG, Watson KD, Lunt M, Hyrich KL, Silman AJ, Symmons DP (2007) Reduction in the incidence of myocardial infarction in patients with rheumatoid arthritis who respond to anti-tumor necrosis factor alpha therapy: results from the british society for rheumatology biologics register. Arthritis Rheum 56:2905-2912

9. Greenberg JD, Kremer JM, Curtis JR, Hochberg MC, Reed G, Tsao P et al (2011) Tumour necrosis factor antagonist use and associated risk reduction of cardiovascular events among patients with rheumatoid arthritis. Ann Rheum Dis 70:576-582

10. Jacobsson LT, Turesson C, Gulfe A, Kapetanovic MC, Petersson IF, Saxne T et al (2005) Treatment with tumor necrosis factor blockers is associated with a lower incidence of first cardiovascular events in patients with rheumatoid arthritis. J Rheumatol 32:1213-1218

11. Kotani K, Miyamoto M, Ando H (2017) The effect of treatments for rheumatoid arthritis on endothelial dysfunction evaluated by flow-mediated vasodilation in patients with rheumatoid arthritis. Curr Vasc Pharmacol 15:10-18

12. Capria A, De Nardo D, Baffetti FR, Barbini U, Violo A, Tondo $\mathrm{T}$ et al (2010) Long-term anti-tnf-alpha treatments reverse the endothelial dysfunction in rheumatoid arthritis: the biological coherence between synovial and endothelial inflammation. Int J Immunopathol Pharmacol 23:255-262

13. Sidiropoulos PI, Siakka P, Pagonidis K, Raptopoulou A, Kritikos H, Tsetis D et al (2009) Sustained improvement of vascular endothelial function during anti-tnfalpha treatment in rheumatoid arthritis patients. Scand J Rheumatol 38:6-10

14. Bosello S, Santoliquido A, Zoli A, Di Campli C, Flore R, Tondi $\mathrm{P}$ et al (2008) Tnf-alpha blockade induces a reversible but transient effect on endothelial dysfunction in patients with long-standing severe rheumatoid arthritis. Clin Rheumatol 27:833-839

15. Bilsborough W, Keen H, Taylor A, O'Driscoll GJ, Arnolda L, Green DJ (2006) Anti-tumour necrosis factor-alpha therapy over conventional therapy improves endothelial function in adults with rheumatoid arthritis. Rheumatol Int 26:1125-1131

16. Mazzoccoli G, Notarsanto I, de Pinto GD, Dagostino MP, De Cata A, D'Alessandro G et al (2010) Anti-tumor necrosis factor-alpha therapy and changes of flow-mediated vasodilatation in psoriatic and rheumatoid arthritis patients. Intern Emerg Med 5:495-500

17. van Eijk IC, Serne EH, Dijkmans BA, Smulders Y, Nurmohamed $M$ (2011) Microvascular function is preserved in newly diagnosed rheumatoid arthritis and low systemic inflammatory activity. Clin Rheumatol 30:1113-1118 
18. Del Porto F, Lagana B, Lai S, Nofroni I, Tinti F, Vitale M et al (2007) Response to anti-tumour necrosis factor alpha blockade is associated with reduction of carotid intima-media thickness in patients with active rheumatoid arthritis. Rheumatology (Oxford) 46:1111-1115

19. van Sijl AM, van Eijk IC, Peters MJ, Serne EH, van der HorstBruinsma IE, Smulders YM et al (2015) Tumour necrosis factor blocking agents and progression of subclinical atherosclerosis in patients with ankylosing spondylitis. Ann Rheum Dis 74:119-123

20. Rueda-Gotor J, Llorca J, Corrales A, Blanco R, Fuentevilla P, Portilla V et al (2016) Carotid ultrasound in the cardiovascular risk stratification of patients with ankylosing spondylitis: results of a population-based study. Clin Exp Rheumatol 34:885-892

21. Vlachopoulos C, Gravos A, Georgiopoulos G, Terentes-Printzios D, Ioakeimidis N, Vassilopoulos D et al (2018) The effect of tnf-a antagonists on aortic stiffness and wave reflections: a meta-analysis. Clin Rheumatol 37:515-526

22. Maki-Petaja KM, Hall FC, Booth AD, Wallace SM, Yasmin BPW et al (2006) Rheumatoid arthritis is associated with increased aortic pulse-wave velocity, which is reduced by anti-tumor necrosis factor-alpha therapy. Circulation 114:1185-1192

23. Mathieu S, Pereira B, Dubost JJ, Lusson JR, Soubrier M (2012) No significant change in arterial stiffness in ra after 6 months and 1 year of rituximab treatment. Rheumatology (Oxford) 51:1107-1111

24. Angel K, Provan SA, Hammer HB, Mowinckel P, Kvien TK, Atar D (2011) Changes in arterial stiffness during continued infliximab treatment in patients with inflammatory arthropathies. Fundam Clin Pharmacol 25:511-517

25. Van Doornum S, McColl G, Wicks IP (2005) Tumour necrosis factor antagonists improve disease activity but not arterial stiffness in rheumatoid arthritis. Rheumatology (Oxford) 44:1428-1432

26. van Gestel AM, Prevoo ML, vant Hof MA, van Rijswijk MH, van de Putte LB, van Riel PL (1996) Development and validation of the european league against rheumatism response criteria for rheumatoid arthritis. Comparison with the preliminary American college of rheumatology and the world health organization/international league against rheumatism criteria. Arthritis Rheum 39:34-40

27. Poliska S, Besenyei T, Vegh E, Hamar A, Pusztai A, Vancsa A et al (2019) Gene expression analysis of vascular pathophysiology related to anti-tnf treatment in rheumatoid arthritis. Arthritis Res Ther 21:94

28. Corretti MC, Anderson TJ, Benjamin EJ, Celermajer D, Charbonneau F, Creager MA et al (2002) Guidelines for the ultrasound assessment of endothelial-dependent flow-mediated vasodilation of the brachial artery: a report of the international brachial artery reactivity task force. J Am Coll Cardiol 39:257-265

29. Soltesz P, Der H, Kerekes G, Szodoray P, Szucs G, Danko K et al (2009) A comparative study of arterial stiffness, flow-mediated vasodilation of the brachial artery, and the thickness of the carotid artery intima-media in patients with systemic autoimmune diseases. Clin Rheumatol 28:655-662

30. Kanters SD, Algra A, van Leeuwen MS, Banga JD (1997) Reproducibility of in vivo carotid intima-media thickness measurements: a review. Stroke 28:665-671

31. Baulmann J, Schillings U, Rickert S, Uen S, Dusing R, Illyes M et al (2008) A new oscillometric method for assessment of arterial stiffness: comparison with tonometric and piezo-electronic methods. J Hypertens 26:523-528

32. Rueda-Gotor J, Llorca J, Corrales A, Parra JA, Portilla V, Genre F et al (2018) Cardiovascular risk stratification in axial spondyloarthritis: carotid ultrasound is more sensitive than coronary artery calcification score to detect high-cardiovascular risk axial spondyloarthritis patients. Clin Exp Rheumatol 36:73-80

33. Gonzalez-Juanatey C, Llorca J, Martin J, Gonzalez-Gay MA (2009) Carotid intima-media thickness predicts the development of cardiovascular events in patients with rheumatoid arthritis. Semin Arthritis Rheum 38:366-371

34. van Sijl AM, Peters MJ, Knol DK, de Vet HC, Gonzalez-Gay MA, Smulders YM et al (2010) Carotid intima media thickness in rheumatoid arthritis as compared to control subjects: a metaanalysis. Semin Arthritis Rheum 40:389-397

35. Kerekes G, Soltesz P, Szucs G, Szamosi S, Der H, Szabo Z et al (2011) Effects of adalimumab treatment on vascular disease associated with early rheumatoid arthritis. Isr Med Assoc J $13: 147-152$

36. Heathfield SK, Parker B, Zeef LA, Bruce IN, Alexander MY (2013) Certolizumab pegol attenuates the pro-inflammatory state in endothelial cells in a manner that is atheroprotective. Clin Exp Rheumatol 31:225-233

37. Shu Q, Amin MA, Ruth JH, Campbell PL, Koch AE (2012) Suppression of endothelial cell activity by inhibition of tnfalpha. Arthritis Res Ther 14:R88

38. Robustillo-Villarino M, Alegre-Sancho JJ, Rodilla-Sala E, Corrales A, Llorca J, Gonzalez-Gay MA et al (2017) Pulse wave velocity and augmentation index are not independently associated with carotid atherosclerosis in patients with rheumatoid arthritis. Clin Rheumatol 36:2601-2606

39. Rueda-Gotor J, Llorca J, Corrales A, Blanco R, Fuentevilla P, Portilla $\mathrm{V}$ et al (2016) Subclinical atherosclerosis is not increased in patients with non-radiographic axial spondyloarthritis. Clin Exp Rheumatol 34:159-160

40. Roodenrijs NMT, de Hair MJH, van der Goes MC, Jacobs JWG, Welsing PMJ, van der Heijde D et al (2018) Characteristics of difficult-to-treat rheumatoid arthritis: results of an international survey. Ann Rheum Dis 77:1705-1709

41. Song BG, Park JB, Cho SJ, Lee SY, Kim JH, Choi SM et al (2009) Pulse wave velocity is more closely associated with cardiovascular risk than augmentation index in the relatively low-risk population. Heart Vessels 24:413-418

\section{Congress citations}

42. Bodnár N, Kerekes G, Végh E, Gulyás K, Balázs F, Pogácsás L, Pál I, Szekanecz Z, Szántó S (2013) Long-term effects of etanercept treatment on vascular function and lipid parameters in ankylosing spondylitis. Ann Rheum Dis 72(Suppl 3):958

43. Végh E, Kerekes G, Váncsa A, Balázs F, Pogácsás L, Pál I, Szántó S, Szücs G, Szekanecz Z (2013) Long-term effects of etanercept and certolizumab pegol treatment on vascular function and lipid parameters in rheumatoid arthritis. Ann Rheum Dis 72(Suppl 3):411

44. Bodnár N, Végh E, Kerekes Gy, Balázs F, Pogácsás L, Szekanecz Z, Szántó S (2013) Vascular effects of etanercept in ankylosing spondylitis [Hungarian]. Hung Rheumatol 54:150

45. Végh E, Balázs F, Kerekes Gy, Pogácsás L, Szántó S, Szekanecz Z, Szúcs G, Váncsa A (2013) Vascular effects of etanercept and certolizumab pegol in rheumatoid arthritis [Hungarian]. Hung Rheumatol 54:175

46. Végh E, Váncsa A, Kerekes G, Balázs F, Pogácsás L, Bodnár N, Szücs G, Szántó S, Szekanecz Z (2014) Long-term effects of etanercept and certolizumab pegol treatment on vascular function and lipid parameters in rheumatoid arthritis and ankylosing spondylitis. Ann Rheum Dis 73(Suppl 1):A36-A37

47. Kerekes G, Balogh E, Végh E, Váncsa A, Csomor P, Pogácsás L, Szántó S, Szúcs G, Szekanecz Z (2015) Effects of anti-TNF therapy on markers of angiogenesis and vascular pathology in arthritis [Hungarian]. Cardiol Hung 45:D95-D96

Publisher's Note Springer Nature remains neutral with regard to jurisdictional claims in published maps and institutional affiliations. 


\section{Affiliations}

\section{Edit Végh ${ }^{1}$. György Kerekes ${ }^{2}$ - Anita Pusztai ${ }^{1}$ - Attila Hamar ${ }^{1}$ Szilvia Szamosi ${ }^{1}$ - Andrea Váncsa ${ }^{1}$. Levente Bodoki ${ }^{1}$. Lilla Pogácsás ${ }^{1}$. Fruzsina Balázs ${ }^{1}$ • Katalin Hodosi ${ }^{1}$ · Andrea Domján ${ }^{1}$ · Sándor Szántó ${ }^{1,3} \cdot$ Zoltán Nagy $^{1}$. Zoltán Szekanecz ${ }^{1}$ Gabriella Szűcs ${ }^{1}$}

\author{
Edit Végh \\ veghe22@gmail.com \\ György Kerekes \\ gkerekesg@gmail.com \\ Anita Pusztai \\ anita.pusztai01@gmail.com \\ Attila Hamar \\ attilahamar.2010@gmail.com \\ Szilvia Szamosi \\ szamosi.szilvi@gmail.com \\ Andrea Váncsa \\ andrea.vancsa@gmail.com \\ Levente Bodoki \\ bodoki.levente@gmail.com \\ Lilla Pogácsás \\ pogacsas.lilla@gmail.com \\ Fruzsina Balázs \\ b.fruzsinadr@gmail.com
}

Katalin Hodosi

khodosi@gmail.com

Andrea Domján

domjan.andrea@gmail.com

Sándor Szántó

szanto.sandor@med.unideb.hu

Zoltán Nagy

dr.nagy.z@gmail.com

Gabriella Szúcs

szucs.gabriella@med.unideb.hu

1 Department of Rheumatology, Institute of Medicine, University of Debrecen, Faculty of Medicine, Nagyerdei str 98, Debrecen 4032, Hungary

2 Department of Angiology, Institute of Medicine, University of Debrecen, Faculty of Medicine, Debrecen, Hungary

3 Department of Sports Medicine, University of Debrecen, Faculty of Medicine, Debrecen, Hungary 